\title{
Corrigendum \\ Endocannabinoids in Chronic Migraine: CSF Findings Suggest a System Failure
}

Paola Sarchielli, Luigi Alberto Pini, Francesca Coppola, Cristiana Rossi, Antonio Baldi, Maria Luisa Mancini and Paolo Calabresi

Neuropsychopharmacology (2007) 32, 1432. doi:10.1038/sj.npp. 1301320

Correction to: Neuropsychopharmacology (2007) 32, 1384-1390. doi:10.1038/sj.npp.1301246

Following the online publication of this article, the authors noted that the affiliations for Dr Baldi and Professor
Calabresi were incorrect. The correct affiliations are: Neurologic Clinic, Department of Medical and Surgical Specialties and Public Health, University of Perugia, Perugia, Italy and IRCCS, Fondazione Santa Lucia, Rome, Italy. 